Check for updates

Cite this: New J. Chem., 2019, 43, 2411

DOI: 10.1039/c9nj90010a

rsc.li/njc

\section{Correction: Multifunctional polyurethanes synthesized from different triarylamine units with electrochromic, photogeneration, memory storage and sensing properties}

\author{
Xu Zhang, ${ }^{a}$ Qingyi Lu, ${ }^{a}$ Caiyu Yang, ${ }^{a}$ Shuo Zhao, ${ }^{a}$ Yan Wang, ${ }^{a}$ Wei Zhang, ${ }^{a}$ \\ Haijun Niu, ${ }^{* a}$ Ping Zhao, ${ }^{b}$ Wen Wang*c and Jinghe Fan ${ }^{a}$
}

Correction for 'Multifunctional polyurethanes synthesized from different triarylamine units with electrochromic, photogeneration, memory storage and sensing properties' by Xu Zhang et al., New J. Chem., 2019, 43, 1177-1185.

The authors would like to correct the Acknowledgements section, as the grant number provided for the National Natural Science Foundation of China is incorrect in the published article. The Acknowledgements section should read:

This work was supported by the National Natural Science Foundation of China (51773053); the Heilongjiang Province Foundation for Returners Overseas (LC2018024); the Reserve Talented Person of Harbin (2015RAXXJ015); and the Open Foundation of the State Key Laboratory of Urban Water Resources and Environment (ES201701).

The Royal Society of Chemistry apologises for these errors and any consequent inconvenience to authors and readers.

\footnotetext{
${ }^{a}$ Key Laboratory of Functional Inorganic Material Chemistry, Ministry of Education, Department of Macromolecular Science and Engineering, School of Chemical, Chemical Engineering and Materials, Heilongjiang University, Harbin 150080, P. R. China. E-mail: haijunniu@hotmail.com

${ }^{b}$ Key Laboratory for Advanced Materials and Institute of Fine Chemicals, East China University of Science and Technology, Shanghai 20037, P. R. China

${ }^{c}$ School of Materials Science and Engineering, Harbin Institute of Technology, Harbin 150080, P. R. China. E-mail: wangwen@hit.edu.cn
} 